\title{
A Content Based Approach to Medical X-Ray Image Retrieval using Texture Features
}

\author{
Sumathi Ganesan ${ }^{*}$ and T.S. Subashini ${ }^{* *}$ \\ Department of Computer Science and Engineering
}

Faculty of Engineering and Technology, Annamalai University, Annamalai Nagar - 608 002, India.

$$
\text { e-mail : sumi.ganesan@yahoo.com* rtramsuba@gmail.com** }
$$

\begin{abstract}
Of late, the amount of digital X-ray images that are produced in hospitals is increasing incredibly fast. Efficient storing, processing and retrieving of X-ray images have thus become an important research topic. With the exponential need that arises in the search for the clinically relevant and visually similar medical images over a vast database, the arena of digital imaging techniques is forced to provide a potential and path-breaking methodology in the midst of technical advancements so as to give the best match in accordance to the user's query image. CBIR helps doctors to compare X-rays of their current patients with images from similar cases and they could also use these images as queries to find the similar entries in the X-ray database. This paper focuses on six different classes of X-ray images, viz. chest, skull, foot, spine, pelvic and palm for efficient image retrieval. Initially the various X-rays are automatically classified into the six-different classes using BPNN and SVM as classifiers and GLCM co-efficient as features for classification. Indexing is done to make the retrieval fast and retrieval of similar images is based on the city block distance.
\end{abstract}

Key Words: Content based image retrieval; back propagation neural network; support vector machine; gray level cooccurrence matrix and city block distance method.

\section{Council for Innovative Research}

Peer Review Research Publishing System

\section{Journal: INTERNATIONAL JOURNAL OF COMPUTERS \& TECHNOLOGY}

Vol 12, No. 7

editor@cirworld.com

www.cirworld.com, www.ijctonline.com 


\section{Introduction}

CBIR techniques could be valuable to radiologists in accessing medical images by identifying similar images in image archives that could assist with decision support. Radiologists always utilize broader patients specific or demographic knowledge such as clinical history or results of other tests, in their decision making process as such. It is expected that decision support system would incorporate these data as well. The role for CBIR in medical applications is potentially very powerful, in addition to enabling similarity based indexing, the framework could provide computer aided diagnostic support based on image content as well as on other meta-data associated with medical images. For example, in mammography Xray interpretation, there is a variation in sensitivity, specificity and area under the receiver operating characteristic curve among radiologists $[1,2]$. Usually, out of the numerous images in the database, radiologists would like to pinpoint the query image against the possible and practical combination of the image-set that not only contains the true ones but also falsepositive and false-negative would be mingled with each other. Hence, the efficacy of the image retrieval system would be greatly dependent on the percentage accuracy of the required image, for which it is being trained.

\section{Previous Work}

In the literature, some approaches for content based retrieval described to support specific medical tasks have been published. In [3] medical applications are one of the priority areas where CBIR can meet more success outside the experimental sphere due to population aging in developed countries. Notwithstanding the progress already achieved in the few frameworks available here is still a lot of work to be done in order to develop a commercial system able to fulfill image retrieval/diagnosis comprehending a broader image domain. In [4] a mammographic database annotated with the IRMA code is presented. This database contains more image direction and anatomical information than the mammographic images. In [5] image retrieval using invariant moments, GLCM and gray-scale resolution for medical images are discussed. The work in [6] suggest for a hierarchical medical image classification method and the scheme proposed was able to provide better accuracy rates semantically. However, the accuracy rates are affected mainly based on the careful hierarchical selection of classes. The work in [7] presents a robust image retrieval methodology for the problem of hidden classes that invariably all the CBIR systems suffer from. As the queries associated with hidden classes cannot be accurately answered using a traditional CBIR system, this proposed method incorporates a new query detection technique and a self-adaptive retrieval strategy that focuses on the augmentation of the image classification and fostering the speed and accuracy of the retrieval process. The work in [8] proposes a strategy for the image retrieval using colour difference histogram $(\mathrm{CDH})$. Unlike the existing histograms, the $\mathrm{CDH}$ counts the perceptually uniform colour difference between two points under different background with regard to colours and edge orientations, whereas the conventional methods merely count the number or frequency of pixels. The work in [9] describes a new colour image segmentation method based on low-level features including colour, texture and spatial information using mean-shift algorithm, wherein some cases were found to be not sufficient for superior segmentation with respect to colour and spatial information. The proposed work in [10] narrates three different scopes for image retrieval using various image feature proposals viz., colour co-occurrence matrix, difference between pixels of scan pattern and colour histogram. An effective image retrieval scheme using colour, texture and shape features that were ultimately projected to have achieved higher retrieval efficiency is given in [11]. A new CBIR approach for biometric security based on colour, texture and shape features e controlled by fuzzy heuristics is described in [12]. Multi-resolution approach for retrieval of rotation invariant texture image is presented in $[13,14]$.

\section{Proposed Work}

The block diagram of the proposed work is shown in Fig. 1.

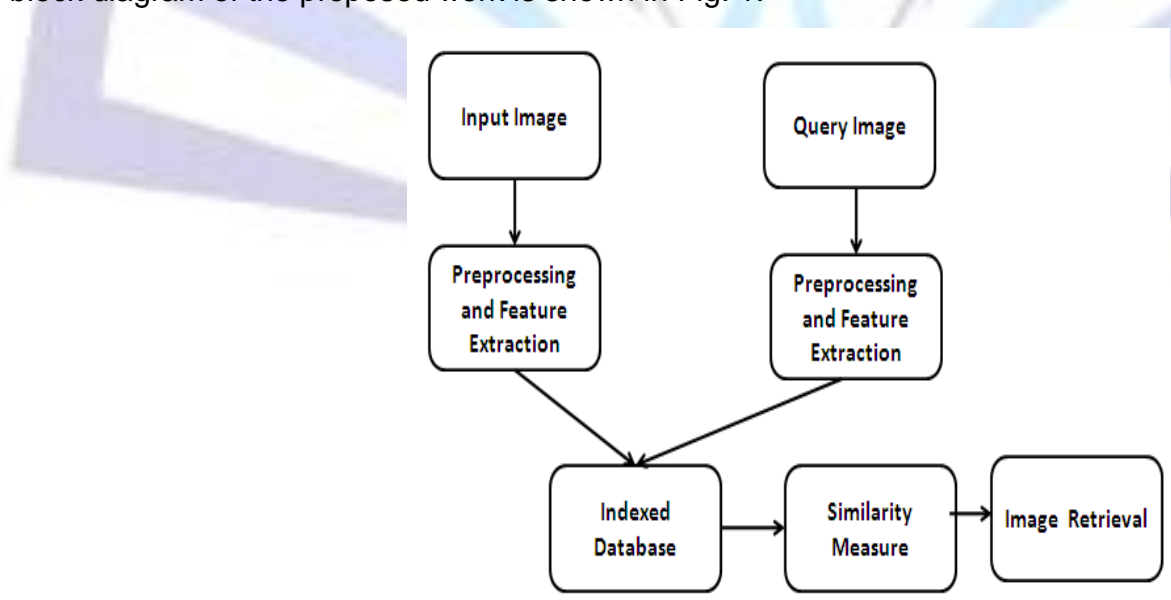

Fig. 1 Block Diagram of the proposed work

\section{Pre-processing and Feature Extraction}

The first phase of the image retrieval methodology initiates with the pre-processing, which is used to reduce the undesired distortions and noise in the X-Ray images, wherein segmentation process is done to find the region of interest (ROI). 
The ROI is found by segmenting the biggest region in the image. Connected Component Labelling (CCL) is applied for this purpose.

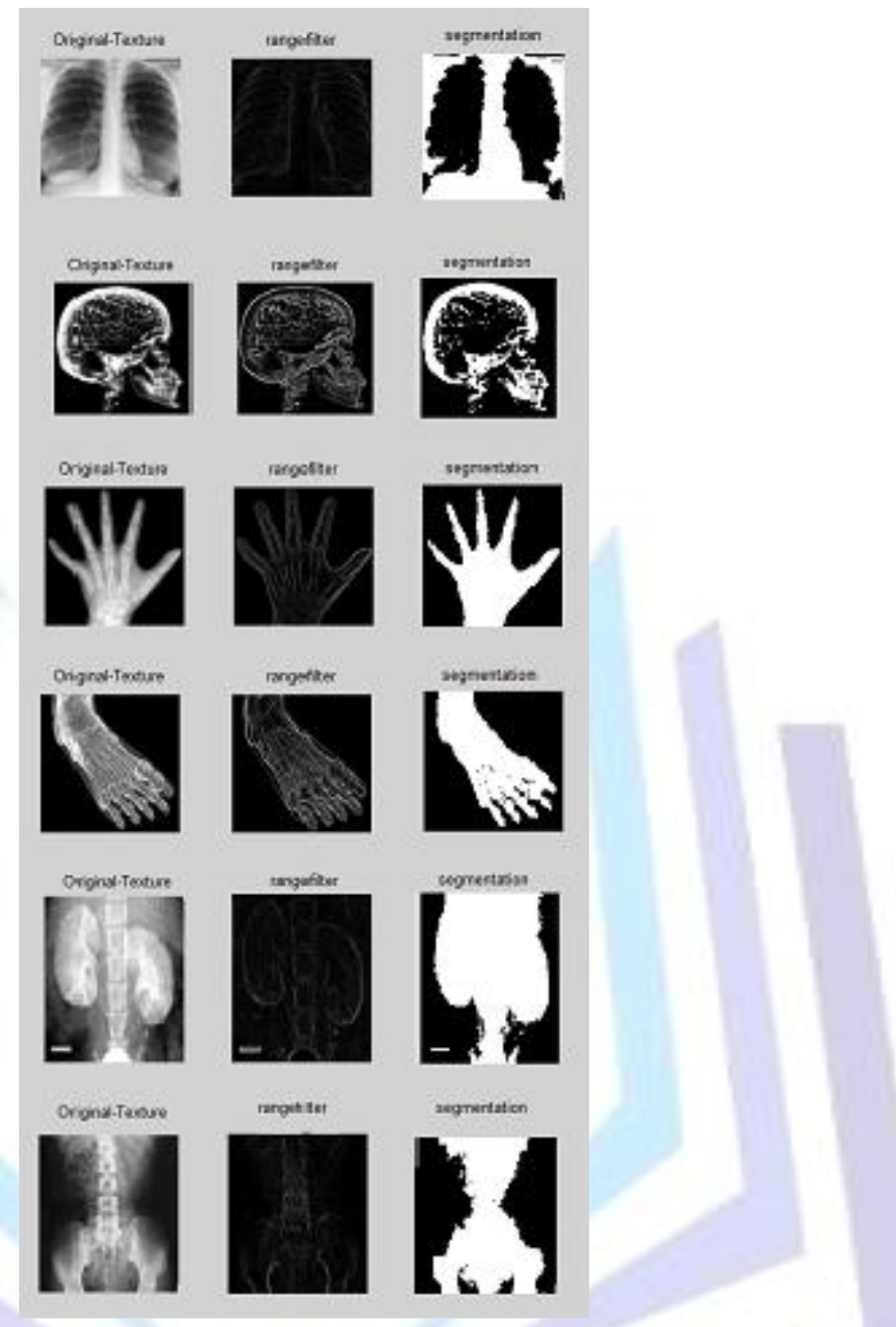

\section{Fig.2 Pre-processing Results}

CCL scans an image and groups its pixels into components based on pixel connectivity, i.e. all pixels in connected component share similar pixel intensity values and are in some way connected with each other. Once all groups have been determined, each pixel is labelled with a gray-level or a color (color labeling) according to the component.

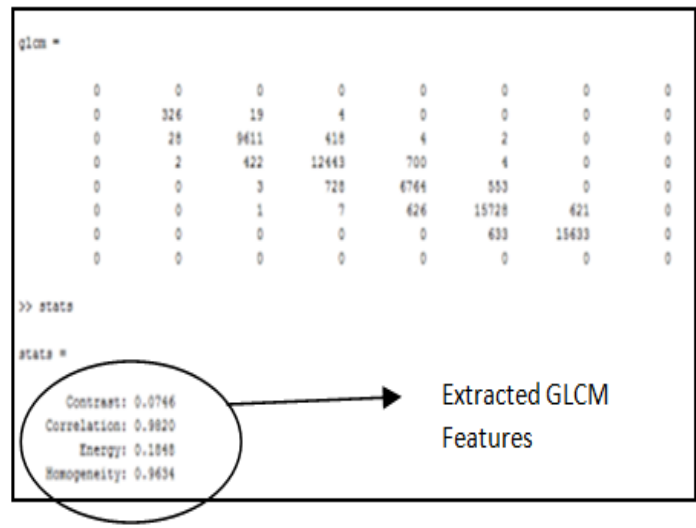

Fig. 3 Extracted GLCM Features 
The results of the pre-processing step are shown in Fig. 2. The first column of Fig. 2 represents the original images; column 2 shows the filtered images and column 3 shows the ROI obtained after retaining the biggest connected component.

Next, image feature textures are extracted using Grey-Level Co-occurrence Matrix (GLCM) and five of the high level GLCM features like energy, entropy, contrast, homogeneity and correlation are fed to the BPNN for classification of $X-$ Ray images. The extracted GLCM features are shown in Fig. 3.

\section{Classification}

Classification is used to classify the X-ray images based on its features. Here back propagation neural network classifier is employed to classify the X-Ray images into any of the six classes of images. The back propagation neural network is the most commonly used neural network in classification applications [15]. It uses a set of interconnected neural elements that process the information in a layered manner. A computational neural element, called perceptron, provides an output as a threshold weighted sum of all inputs. The basic function of the perceptron is shown in Fig. 4 is analogous to the synaptic activities of a biological neuron [16]. In a layered network structure, the neural element may receive its input from an input vector or other neural elements. A weighted sum of these inputs constitutes the argument of a non-linear activation function such as a sigmoidal function. The resulting threshold value of the activation function is the output of the neural element. The output is distributed along weighted connections to other neural elements. The computational output of a neural element

can be expressed in the following equation:

$$
y=F\left(\sum_{i=1}^{n} w_{i} x_{i}+w_{n+1}\right)
$$

where $\mathrm{F}$ is a non-linear activation function that is used to threshold the weighted sum of inputs $x i$ and wi is the respective weight [17].

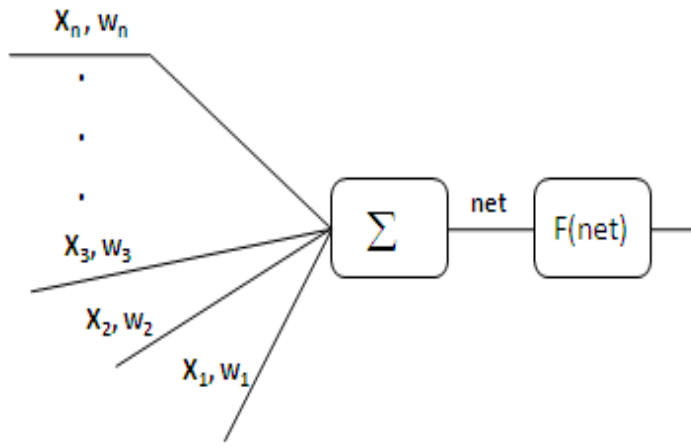

Fig. 4. A computational Neural Model

A three layer network is used to classify and the performance of the classifier is checked for different network structure and the best performance is achieved with the network structure having 18 input neurons and 14 hidden neurons. The most popular classifier Support Vector Machine (SVM) is then deployed to yield the results of the most relevant images out of the query image and for which the network is trained. The typical overview of SVM is as Fig. 4.1. The inherent property of the SVM gives a set of output data that match with that of the input, being arrived at the state-of-the-art prediction and analysis from the possible classes of the images. Thus, SVM classifier proves to be more beneficial to the radiologists when compared with that of the BPNN and their calculated numerical values of accuracy are shown in table 1.

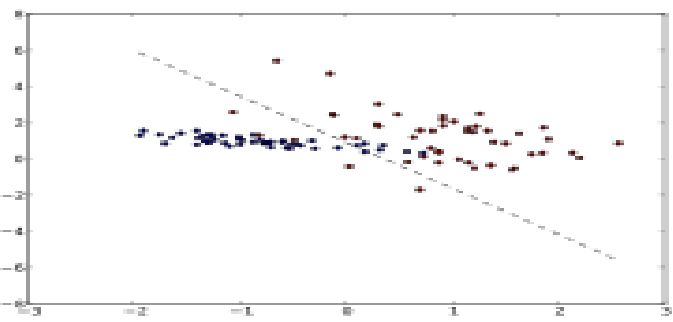

Fig. 4.1 Overview of SVM

The performance of the classifier is calculated as given by the metrics; 
Precision $=\frac{T P}{T P+F P}$

Recall $=\frac{T P}{T P+F N}$

Accuracy $=\frac{T P+T N}{T P+T N+F P+F N}$

\section{Feature Database Creation}

For this study, 250 images each of the six class of X-rays namely chest, skull, spine, foot, pelvic and palm are taken. The block diagram for feature database creation is shown in Fig. 4

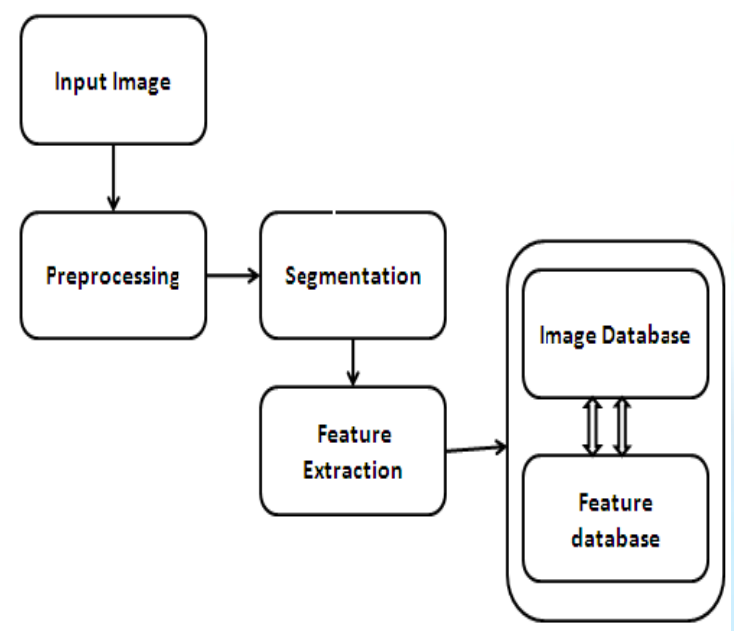

Fig.5 Feature Database Creation

All the images are subjected to pre-processing, segmentation, classification and on the basis of the class label they are indexed into any one of the six databases created for each of the class. The X-Ray image along with its corresponding feature vector is stored in the database and the user interface created for this work is shown in 6 . Fig. 7 shows the feature extracted and stored in a database.

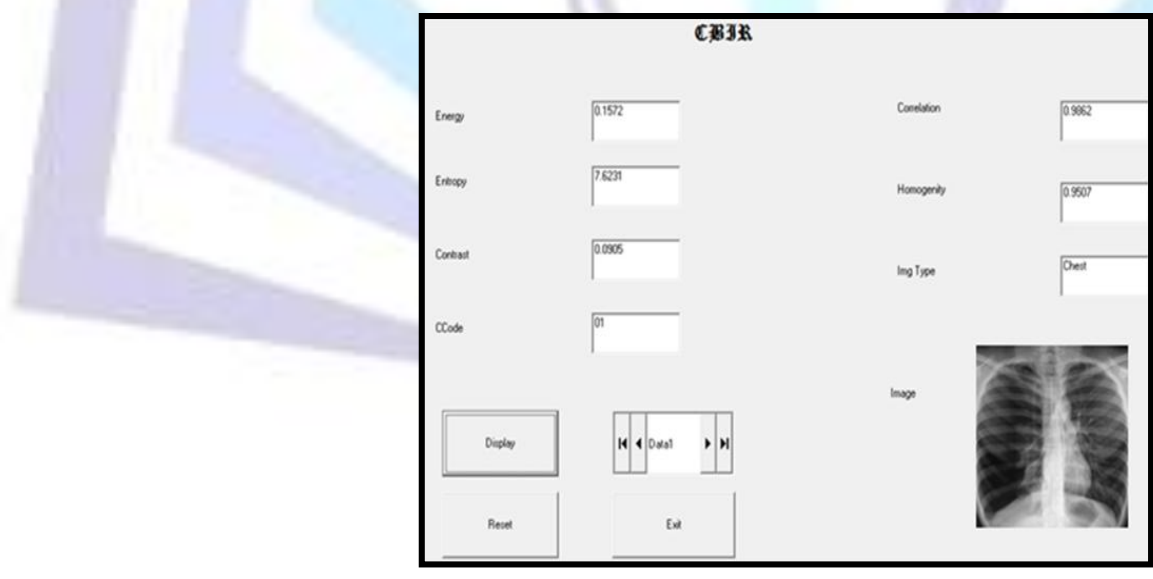

Fig. 6 GUI of the proposed work 


\begin{tabular}{|c|c|c|c|c|c|c|}
\hline \multicolumn{7}{|l|}{ HDB } \\
\hline 10 & Image & Energy & Entropy & Contrast & - Correlation. & Homogenit) - \\
\hline 1 & Package & 0.1547 & 6.4957 & 0.2706 & 0.9684 & 0.9113 \\
\hline 2 & Package & 0.1804 & 7.2009 & 0.0979 & 0.9797 & 0.9515 \\
\hline 3 & Package & 0.1874 & 6.4358 & 0.5935 & 0.9408 & 0.8804 \\
\hline 4 & Package & 0.1238 & 7.3013 & 0.1277 & 0.9850 & 0.9379 \\
\hline 5 & Package & 0.1334 & 7.0111 & 0.1656 & 0.9791 & 0.9299 \\
\hline 6 & Package & 0.2261 & 6.1834 & 0.2865 & 0.9672 & 0.8933 \\
\hline 7 & Package & 0.1373 & 7.3774 & 0.1712 & 0.9768 & 0.9211 \\
\hline 8 & Package & 0.1580 & 6.5469 & 0.2003 & 0.9562 & 0.9158 \\
\hline 9 & Package & 0.1830 & 7.2287 & 0.1113 & 0.9684 & 0.9470 \\
\hline 10 & Package & 0.1730 & 6.9013 & 0.0995 & 0.9884 & 0.9521 \\
\hline 11 & Package & 0.1806 & 6.7744 & 0.0957 & 0.9852 & 0.9544 \\
\hline 12 & Package & 0.1211 & 7.2848 & 0.2234 & 0.9840 & 0.9097 \\
\hline 13 & Package & 0.1383 & 6.6878 & 0.2294 & 0.9569 & 0.9118 \\
\hline 14 & Package & 0.1806 & 6.8394 & 0.1799 & 0.9821 & 0.9321 \\
\hline 15 & Package & 0.1593 & 7.0755 & 0.1288 & 0.9802 & 0.9386 \\
\hline 16 & Package & 0.1335 & 6.9853 & 0.1639 & 0.9836 & 0.9348 \\
\hline 17 & Package & 0.1260 & 7.6534 & 0.1720 & 0.9851 & 0.9183 \\
\hline 18 & Package & 0.1640 & 6.5394 & 0.1330 & 0.9869 & 0.9381 \\
\hline 19 & Package & 0.1485 & 6.7599 & 0.0998 & 0.9904 & 0.9515 \\
\hline 20 & Package & 0.1528 & 7.2642 & 0.0805 & 0.9929 & 0.9598 \\
\hline
\end{tabular}

Fig. 7 Database Creation

\section{Image Retrieval}

Given a query image, the proposed system retrieves the nearest three similar query images based on the City block distance method. The block diagram for the retrieval is shown in Fig. 8. The query image is subjected to preprocessing, segmentation, classification and the resultant class label is used to index into the feature database. City block distance method is used to retrieve the top most three feature vectors based on the feature vector of the query image. The X-ray images corresponding to the most relevant three feature vectors are retrieved from the image database and displayed.

The distance between two image feature vectors is calculated by using the following expression;

The City block distance between $\left(\boldsymbol{x}_{1}, \boldsymbol{x}_{2}\right)$ and $\left(\boldsymbol{y}_{1}, \boldsymbol{y}_{2}\right)$ is

where,

$$
D=\left|x_{1}-x_{2}\right|+\left|y_{1}-y_{2}\right|
$$

$D$ is the City block distance.

$\left(x_{1}, x_{2}\right)$ is the position of query image.

$\left(\boldsymbol{y}_{1}, \boldsymbol{y}_{2}\right)$ is the position of the database image.

Table : 1 Comparison between BPNN \& SVM Classifiers

\begin{tabular}{|l|l|l|l|}
\hline $\begin{array}{l}\text { Sl. } \\
\text { No. }\end{array}$ & $\begin{array}{l}\text { Feature } \\
\text { Extraction }\end{array}$ & Classifier & Accuracy \\
\hline 1 & GLCM & BPNN & $88.33 \%$ \\
\hline 2 & GLCM & SVM & $92.82 \%$ \\
\hline
\end{tabular}

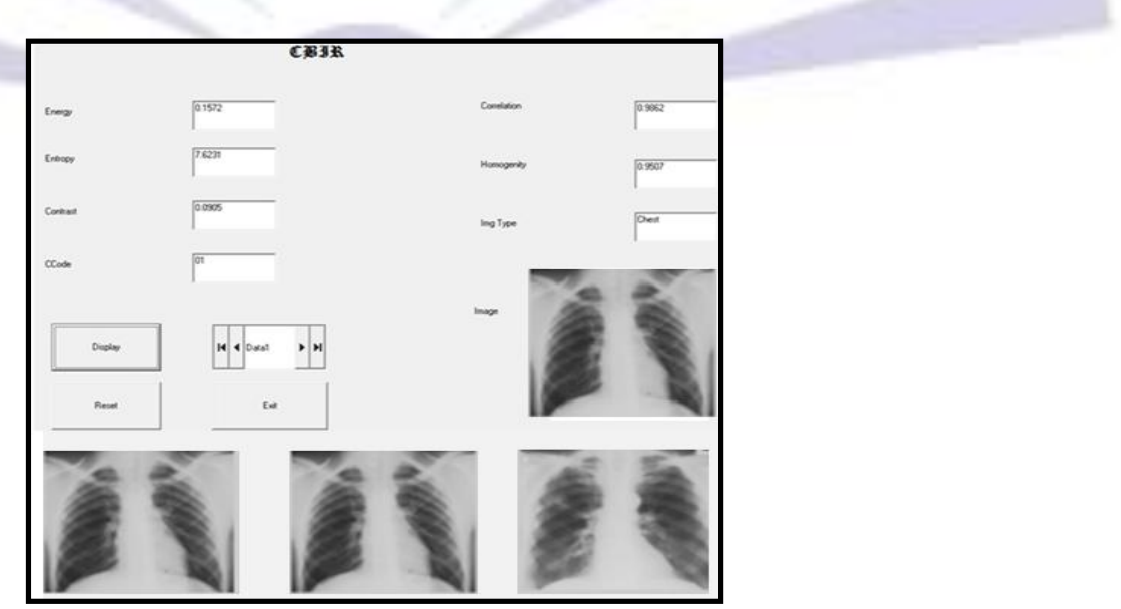

Fig.9 Image Retrieval 


\section{Conclusion}

In this work, an attempt has been made to retrieve similar X-ray images based on the content of the query image. This paper focuses on six different classes of X-ray images namely chest, skull, spine, foot, pelvic and palm. Preprocessing of X-Ray images for noise reduction and enhancement is done by average filter and connected component labelling is carried out to segment the region of interest. Image texture features were extracted using Grey level Cooccurrence matrix (GLCM) and the five most prominent GLCM features are fed to the BPNN classifier which gave a classification accuracy of $88.33 \%$ and that of SVM for $92.82 \% \%$. After classification, a database is created for each of the six classes of X-ray images using the result of SVM classifier, owing to its higher accuracy. City block distance method is used as the metric for retrieving the top three similar images from the image database based on the feature vector of the query image. This results so obtained shows that this system can be effective in content based image retrieval of $X$-ray images and will be helpful for the radiologists in retrieving similar cases as same as the query image.

\section{References}

1. John Eakins and Margaret Graham, Content Based Image Retrieval, University of Northumbria at Newcastle, Oct. 1999.

2. Stephen T.C. Wong, Medical Image Databases,

3. C. Akgul, D. Rubin, S. Napel, C. Beaulieu, H. Greenspan and B. Acar, "Content Based Image Retrieval in Radiology: Current Status and Future Directions", Journal of Digital Imaging, [Epud ahead of print], 2010

4. J.E.E. Oliveira, A.P.B. Lopes, G. Camara-Chavez, A. de Aroujo and T.M. Deserno, "MammoSVD: A Content Based Image Retrieval system using a refernce database of mammographies" Computer Based Medical Systems, 2009. CBMS 2009. 22nd IEEE international Symposium on 2009.

5. Ramamurthy, K. R. Chandran, S. Aishwarya, and P.Janaranjani, "CBMIR: Content Based Image Retrieval using Invariant Moments, GLCM and Gray scale Resolution for Medical Images”, European Journal of Scientific Research, ISSN 1450-216X ol.59 No.4 (2011),pp. 460-471

6. Hossein Pourghassem, Hassan Ghassemian, Content based medical image classification using a new hierarchical merging scheme, Computerized Medical Imaging and Graphics, Elsevier, 32 (2008),651-661.

7. Jun Zhang, Lei Ye, Yang Xiang, Wanlei Zhou, Robust image retrieval with hidden classes, Computer Vision and Image Understanding, Elsevier, 117 (2013) 670-679.

8. Guang-Hai Liu a,b,n, Jing-YuYang, Content-based image retrieval using color difference histogram, Pattern Recognition, Elsevier, 46 (2013) 188-198.

9. Mustafa Ozden, Ediz Polat, A color image segmentation approach for content-based image retrieval, Pattern Recognition, Elsevier, 40 (2007) 1318 - 1325.

10. Chuen-Horng Lin, Rong-Tai Chen, Yung-Kuan Chan, A smart content-based image retrieval system based on color and texture feature, Image and Vision Computing, Elsevier, 27 (2009) 658-665.

11. Xiang-Yang Wang, Yong-Jian Yu, Hong-Ying Yang, An effective image retrieval scheme using color, texture and shape features, Computer Standards \& Interfaces, Elsevier, 33 (2011) 59-68.

12. Kashif Iqbal, Michael O. Odetayo, Anne James, Content-based image retrieval approach for biometric security using colour, texture and shape features controlled by fuzzy heuristics, Journal of Computer and System Sciences, Elsevier, 78 (2012) 1258-1277.

13. V.Ramalingam, B. Palaniappan, N. Panchanatham,S.Palanivel, Measuring advertisement effectiveness - a neural network approach, Expert Systems with applications, Elsevier, vol. 31, no. 1, pp. 159-163, 2006

14. R. Krishnamoorthi, S. Sathiya devi, A multiresolution approach for rotation invariant texture image retrieval with orthogonal polynomials model, J. Visual Commun. Image Represent. 23 (1) (2012) 18-30.

15. R. O. Duda, P. E. Hart, and D. G. Stork, Pattern Classification, John Wiley \& Sons, Singapore, 2003

16. T.S.Subashini, V. Ramalingam, S. Palanivel, "Automated assessment of breast tissue density in digital mammograms", in International Journal of Computer Vision and Image Understanding (Elsevier), no.114, pp. 33-43, 2010.

17. B. Yegnanarayana, Artificial neural networks, Prentice Hall of India, New Delhi, 1999. 